Musées, Patrimoine et Culture scientifiques et techniques

$158 \mid 2015$

mars-avril 2015

\title{
La muséologie critique : entre ruptures et réinterprétations
}

Jesus Pedro Lorente et Nicole Moolhuijsen

\section{OpenEdition \\ Journals}

Édition électronique

URL : http://journals.openedition.org/ocim/1495

DOI : $10.4000 /$ ocim. 1495

ISSN : 2108-646X

Éditeur

OCIM

Édition imprimée

Date de publication : 1 mars 2015

Pagination : 19-24

ISSN : 0994-1908

Référence électronique

Jesus Pedro Lorente et Nicole Moolhuijsen, «La muséologie critique : entre ruptures et

réinterprétations », La Lettre de I'OCIM [En ligne], 158 | 2015, mis en ligne le 01 mars 2016, consulté le

30 avril 2019. URL : http://journals.openedition.org/ocim/1495 ; DOI : 10.4000/ocim.1495

Ce document a été généré automatiquement le 30 avril 2019.

Tous droits réservés 


\title{
La muséologie critique : entre ruptures et réinterprétations
}

\author{
Jesus Pedro Lorente et Nicole Moolhuijsen
}

Installation en hommage à la muséologue Grete Mostny à l'entrée du musée d'Histoire naturelle de Santiago du Chile.

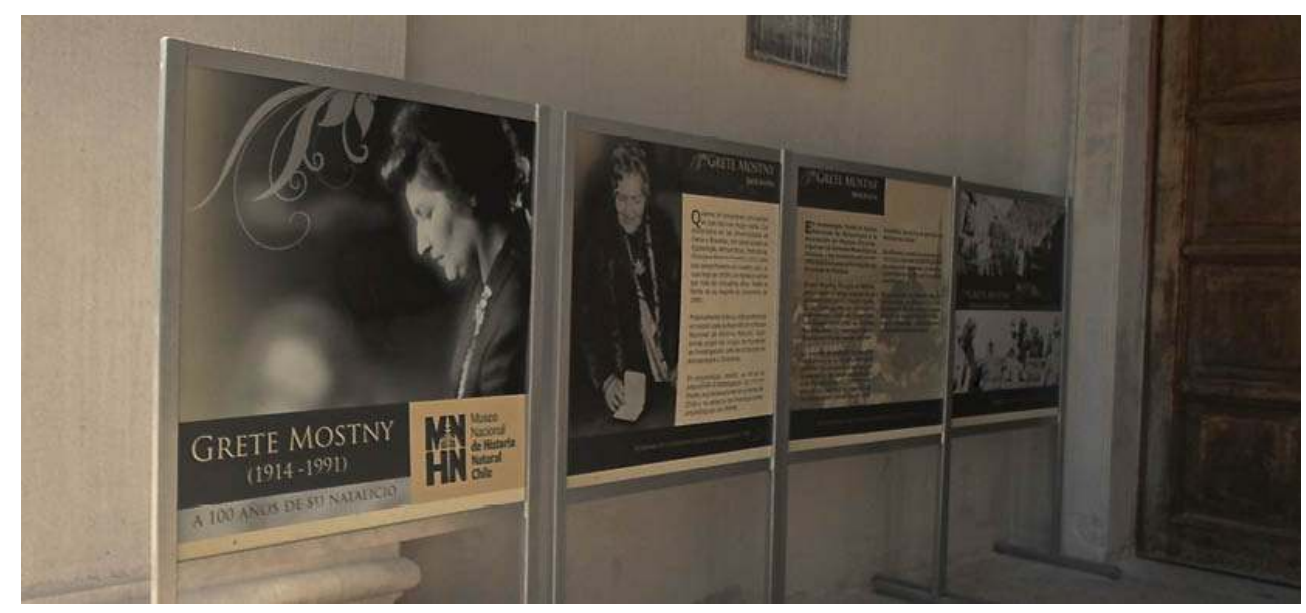

() Jesús Pedro Lorente

1 La muséologie critique est née dans les milieux académiques et particulièrement dans les musées universitaires, choisissant une posture d'analyse critique en réaction aux modes traditionnels de production du discours d'exposition et d'interprétation des collections, et visant à renouveler et transformer les pratiques muséographiques. Les professionnels se revendiquant de ce courant encouragent l'abolition des parcours linéaires, préférant les propositions qui questionnent le public, ainsi que l'utilisation de tous les dispositifs qui favorisent une approche des objets d'exposition à la fois subjective, interactive et participative. Ils invitent les musées à présenter des interprétations personnelles, entrelaçant divers domaines de savoirs, à favoriser des discours pluriels et critiques, et à impliquer activement le public dès la conception et la planification des accrochages ${ }^{1}$. 
2 Les lignes qui suivent exposent quelques caractéristiques de la muséologie critique à travers une sélection d'exemples récents pour montrer comment ce courant se manifeste dans la pratique actuelle de certains musées, mais aussi par un questionnement centré sur le public. Comment renforcer l'importance sociale du musée pour le transformer en un lieu ouvert au dialogue et à la participation ${ }^{2}$ ? Que faire pour qu'un public large et varié puisse se trouver en condition de vivre une expérience de visite ancrée dans le présent et les impliquant? On s'accorde à considérer que dans la culture postmoderne telle qu'elle s'est esquissée depuis une trentaine d'années, il est indispensable de repenser le discours des musées à partir de deux directions : permettre aux visiteurs d'en savoir plus sur les débats d'actualité, mais aussi les inciter à mettre en relation leur propre expérience avec ce qui est exposé. C'est le musée tout entier qui doit s'engager dans une confrontation constante avec l'environnement extérieur et sa communauté, en instaurant un dialogue critique avec ses publics. Et au-delà de ce dialogue critique, il doit mettre en discussion la signification même de ses collections ainsi que l'autorité de ceux qui les gèrent et fabriquent, de facto, le seul discours autorisé sur les collections exposées. En somme, il s'agit ni plus moins de remettre en cause le rôle de chacun et par conséquent la hiérarchie des savoirs entre professionnels et publics. Ce sont là les questions essentielles qui ont été introduites dans le monde des musées par la muséologie critique.

\section{Du récit autoritaire linéaire au discours ouvert à la discussion}

3 Les développements de la philosophie postmoderne et, en particulier, les écrits de Michel Foucault sur les systèmes qui gouvernent la formation de la connaissance, ont bouleversé la conception des disciplines sociales et humaines et notamment les museum studies. C'est dans un tel contexte que l'idée d'objet polysémique tel que Jacques Derrida l'a entre autres conceptualisé, constitué de plusieurs niveaux de signification, est dorénavant incompatible avec une présentation selon un point de vue unique ${ }^{3}$.

Le musée ne peut donc plus être perçu comme un lieu où le vrai est énoncé ou présenté selon un seul domaine du savoir; il doit plutôt se faire porte parole des différentes manières de voir et dénoncer la nature subjective du discours qu'il propose et impose. C'est pour ces raisons que plusieurs musées ont remis en cause les principes qui gouvernent l'arrangement et l'interprétation traditionnels des objets de musée. Et pour cela, ils ont privilégié des discours ouverts, délibérément plus accessibles, dans des accrochages qui réfutent la tradition. Ce faisant, ils mettent en évidence l'épistémologie implicite de la signification qu'ils récusent. Pour la remplacer, ils racontent des histoires différentes et proposent aux visiteurs des questions ouvertes, répondant ainsi à la demande d'un discours pluriel et critique. Une telle approche n'est pas nouvelle, et de nombreux musées, proches des courants de la nouvelle muséologie (comme de nombreux écomusées) ou de la muséologie de la rupture (le musée d'Ethnographie de Neuchâtel), l'avaient très largement développée. C'est dans cette même optique, et souvent sous la dénomination de muséologie critique, que se poursuit cette réflexion à travers le monde. 


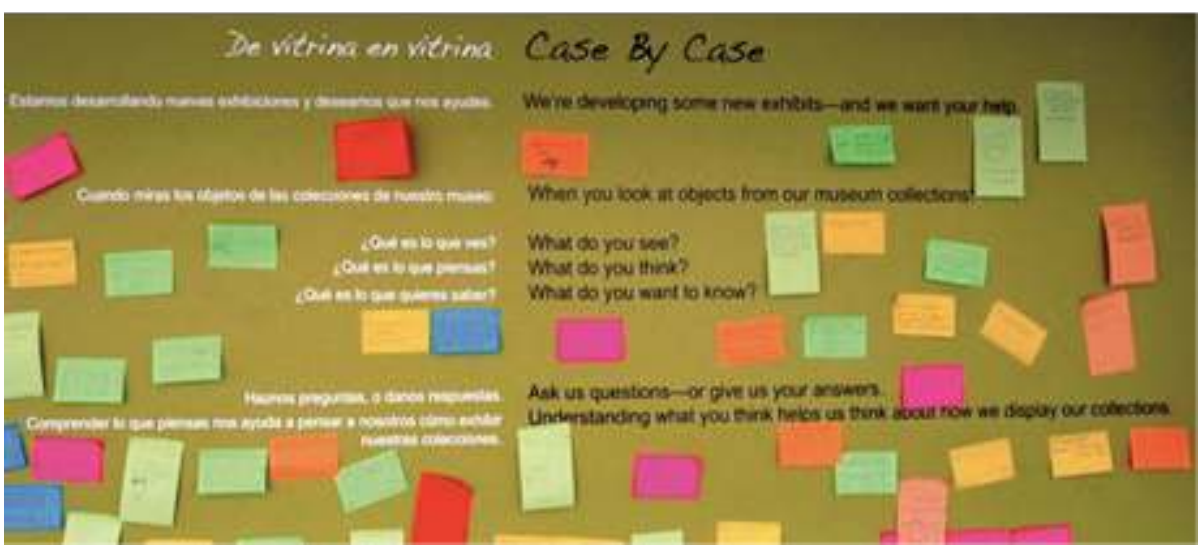

(c) Michael Wall

Case by Case ( Cas par cas ») est le titre d'un projet conduit par le musée d'Histoire naturelle de San Diego en 2011, ayant pour objectif d'améliorer l'accrochage de la collection et le contenu des textes d'accompagnement, à partir des contributions des visiteurs. Au départ, il s'agissait d'une simple expérience participative dans laquelle les objets d'une exposition temporaire étaient momentanément accompagnés de feuilles blanches et de post-it colorés en lieu et place des habituels cartels explicatifs. Il était suggéré au public de répondre sur les post-it à des questions telles que : «Que voyez-vous lorsque vous regardez ces objets? Qu'est ce que vous aimeriez savoir? [...] Comprendre comment ce que vous pensez influence notre manière de voir et d'exposer les collections ». Cette démarche participative, facilement reproductible grâce à son faible coût de réalisation, a permis au musée d'évaluer son dispositif et d'obtenir des informations sur les réactions de son public pour un prix défiant toute concurrence. Les feed-back recueillis ont en outre conduit les membres du personnel à présenter les objets de manière différente, à utiliser des notions qu'ils n'auraient autrement pas prises en considération et, surtout, à prendre conscience de la pluralité des points de vue possibles.

6 En rendant visible la contribution du public lors de la préparation de la muséographie, ce sont les logiques de représentation habituellement dominantes dans les musées qui sont inversées. La réinterprétation et la multiplicité des points de vue sont ainsi encouragées, plutôt que la répétition univoque d'une même histoire. Cette opération de transparence permet aux visiteurs de comprendre que les collections sont sujettes à interprétation, que le personnel du musée projette ses propres connaissances sur les objets et que l'organisation de l'accrochage n'est qu'une proposition parmi d'autres possibles.

7 Le musée Zoologique de Rome est aujourd'hui exemplaire en ce sens. En effet, il traite le thème de la biodiversité et de l'évolution, mais plutôt que de présenter et d'expliquer les sujets du point de vue scientifique conventionnel, les panneaux parlent aux visiteurs de la vie d'aujourd'hui, en intégrant à l'exposition d'animaux naturalisés des objets de la vie quotidienne. À travers une série de questionnements qui se présentent sous la forme d'un quiz ludique adressé aux visiteurs, le comportement des animaux est mis en relation avec celui des êtres humains et les visiteurs sont guidés le long d'un parcours de découverte, à la fois scientifique et personnel, qui suscite le dialogue et l'introspection. 
Une telle contextualisation encourage les raisonnements complexes et la recherche de relations au-delà des frontières classificatrices des disciplines ${ }^{4}$. La typologie des questions posées au public positionne ce dernier en contact direct avec les thèmes de l'exposition, mais sans le filtre du regard du scientifique spécialisé, activant ainsi une curiosité nouvelle laissant place à de multiples interprétations. Le processus d'apprentissage devient ainsi une expérience de construction de sens - plutôt que de transfert -, qui corrobore la contribution constructiviste de la muséologie et valorise le bagage personnel de chaque visiteur ${ }^{5}$.

Le musée dans le musée au museo Enrico Caruso, Florence

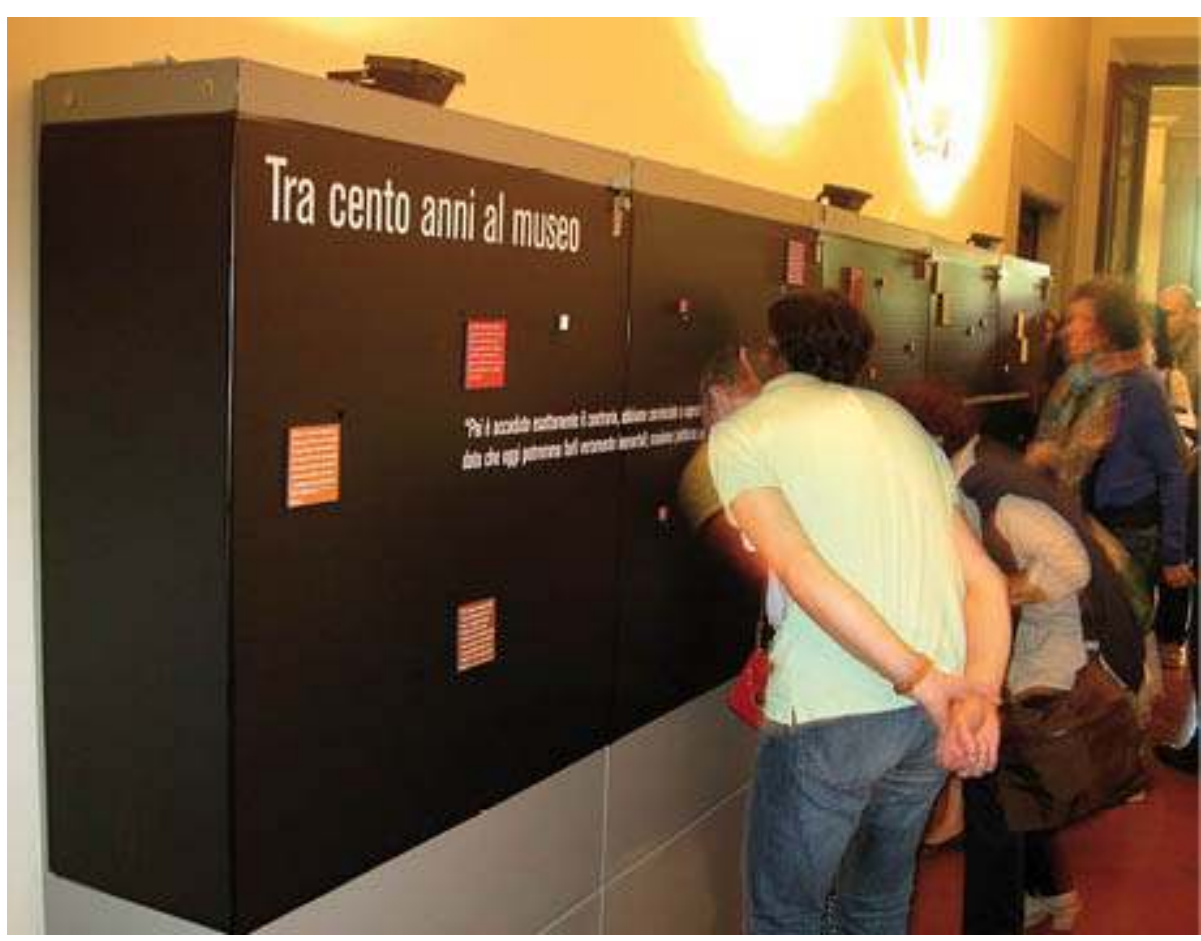

(c) Claudio Rosati

Avec cette nouvelle orientation, c'est la notion de participation elle-même qui est remise en cause dans le contexte muséal. Faire participer, ce n'est plus seulement intégrer à l'exposition des dispositifs dits interactifs, mais au contraire mettre en œuvre un partage de compétences et donner ainsi aux visiteurs la possibilité d'exercer un rôle actif dans la construction d'un savoir personnalisé. Traditionnellement, l'institution positionnait les professionnels des musées comme les seuls dépositaires du savoir et, donc, les seuls susceptibles d'interpréter les collections. Ces modèles doivent aujourd'hui être remis en question, en impliquant le public et la communauté durant les phases d'élaboration, en adoptant des techniques plus expérimentales et en convoquant différents savoirs, le tout à la faveur d'une muséologie (critique) qui redonne du crédit aux valeurs de transparence, d'ouverture culturelle et d'intégration sociale. Le public se voit ainsi propulsé au rang de co-commissaire de l'exposition ou co-conservateur des collections!

C'est grâce à une simple question : "Qu'est-ce qui sera important dans cent ans ? », que le musée Enrico Caruso situé à Florence a transformé une section du musée en un accrochage participatif qui a directement impliqué le public durant toutes les phases de sa réalisation. Au départ, la mise en place d'un caddie à l'entrée du parcours de visite, 
dans le contexte d'un musée consacré à la personnalité du célèbre ténor, a immédiatement décontenancé les visiteurs et suscité leur attention. Les textes d'accompagnement les invitaient à réfléchir au changement de la valeur des objets au cours du temps : ils étaient invités à déposer dans le chariot les objets qui, selon eux, auront acquis de l'importance d'ici cent ans. Les participants ont ainsi été engagés dans un projet destiné à utiliser les espaces du musée comme un espace de création de nouvelles relations entre les personnes et les choses. Le résultat de cette collecte pourrait être considéré comme un exemple de création d'un musée dans le musée. En définitive, les objets proposés et choisis par les visiteurs ont été exposés dans une des salles, accompagnés de textes qu'ils avaient eux-mêmes rédigés. Ce qui fait que cette proposition contributive des visiteurs construit une nouvelle clé de lecture tout à fait intéressante de la collection entière.

11 Ainsi, la mise au jour du processus de muséalisation met en relief la manière dont se produit le changement de valeur d'un objet, en l'inscrivant dans une nouvelle relation de sens qui dépend de l'époque, de l'espace et surtout, de la mentalité de la personne qui l'interprète. De cette manière, le public est encouragé à observer le musée tout entier avec un œil critique, puisqu'il est à même de prendre en compte son propre statut de «créateur» d'histoire. Bien que la participation des visiteurs ne regarde que l'interprétation d'objets d'usage commun, cet exemple met en lumière les modalités à travers lesquelles le savoir est construit dans les coulisses du musée. Et elle permet de discuter le rôle dominant de l'expert dans la formation du discours habituel de l'exposition.

\section{Pour une muséologie des questions sensibles}

Le quatrième numéro de la revue espagnole Museo y Territorio, paru à la fin de 2011, a publié des contributions importantes sur la muséologie critique ${ }^{6}$. Plusieurs articles insistent en effet sur le devoir du musée de mettre en avant son rôle social et éthique, à travers une programmation particulièrement engagée sur les thèmes comme les droits humains et l'intégration des citoyens défavorisés. Il est souvent difficile de vouloir aborder des thèmes sensibles et controversés: il faut du courage pour aborder des thématique politiques, morales ou sociales plutôt que seulement culturelles. Ces thèmes sont généralement considérés comme accessoires par rapport à la nature culturelle du musée, d'autant que ce déplacement en dehors de leur domaine d'expertise apparaît à certains commissaires d'exposition comme une menace. 
Dans la galerie Who am I ? au Science Museum de Londres, le questionnement instaure un dialogue avec le visiteur.

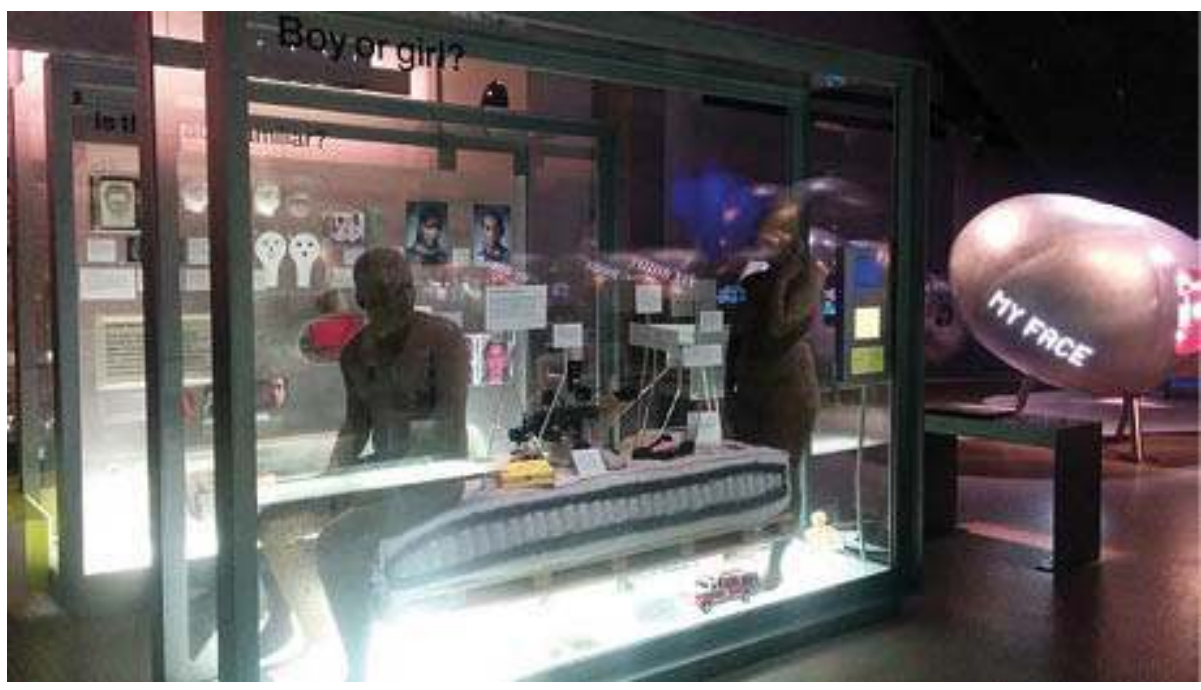

(c) Science Museum

13 À ce propos, il est intéressant d'étudier le cas de la "Who am I gallery? (littéralement : «Galerie Qui suis-je?») du Science Museum de Londres. Ce musée a créé en 2010, à l'occasion du dixième anniversaire du premier séquençage du génome humain, un nouvel accrochage sur le thème du corps et de la personnalité. Le sujet est développé à travers quatre galeries interactives, dont une qui s'attache au thème de l'identité de genre. L'un des objectifs du musée était en effet de sensibiliser les visiteurs à la diversité des genres, en soutenant l'intégration sociale des personnes transgenres. Toutefois, plutôt que de véhiculer ce principe en le présentant comme juste, en soi, moralement, le musée a choisi de poser des questions directes aux visiteurs telles que : "Qu'est ce qui constitue votre genre ? ", « En quoi les garçons et les filles sont-ils différents ? », ou bien « Le genre est-il congénital ? ». Les questions aiguisent l'attention des visiteurs en les invitant à construire leur propre identité de genre en se reconnaissant (ou non) dans les objets exposés. Le genre est ainsi présenté comme une performance inscrite dans un contexte social et culturel, au sein duquel chacun peut trouver sa place, sans pour autant devoir correspondre aux étiquettes «masculin » ou " féminin » qui relèvent, quant à elles, du sexe biologique. De cette manière, la différence entre le concept de sexe, soit le patrimoine génétique, et celui de genre, qui correspond lui au patrimoine culturel, est formulée de manière explicite. Lors de cette expérience, le visiteur est encouragé à dépasser la vision bipolaire du genre («masculin» opposé à «féminin») pour le considérer comme un éventail plus large de possibilités, intégrant les transgenres. De manière générale, l'utilisation du questionnement peut faciliter l'interprétation de thèmes complexes en instaurant un dialogue avec le visiteur en lieu et place de l'affirmation péremptoire, propre au milieu muséal traditionnel. L'exemple du Science Museum de Londres, fruit des efforts des commissaires, des professionnels des services pédagogiques, et autres facilitateurs, montre qu'il est possible d'aborder un sujet controversé ou de mettre en avant un objectif socialement engagé sans que cela signifie pour autant un renoncement des commissaires à l'expertise, ou un refus d'assumer la mission du musée. 

la volonté d'incarner la mission publique et sociale du musée. Le musée se présente ainsi comme un espace ouvert dans lequel il devient, non seulement possible, mais aussi souhaitable, d'aborder des sujets délicats ou potentiellement controversés ${ }^{8}$. Tout cela implique, d'une part, une remise en cause permanente des discours proposés et de la hiérarchie des rôles qui en découle, et d'autre part, une mixité entre pluralisme et volonté de briser les cadres. Aucun professionnel des musées ne devrait jamais renoncer à cette politique.

Cet état d'esprit suppose peut-être une vision moins complaisante qui est plus facile à envisager en période de crise idéologique, comme cela a pu se passer dans de nombreux musées d'histoire culturelle des anciens pays communistes confrontés à des changements radicaux ${ }^{9}$. Par exemple, dans le musée de la Slovaquie de l'Est à Košice, le parti pris dominant d'une histoire sociale à caractère marxiste a été complété par d'autres muséographies. Ainsi, dans la première salle de l'entrée, on montre dorénavant certaines pièces des anciennes collections dans les vitrines du XIXe siècle récupérées dans les réserves, afin d'évoquer l'époque de son fondateur, Imrich Henszlmann. C'est une façon de restaurer la mémoire d'anciens accrochages mais surtout, c'est la pluralité de récits qui est ainsi exhibée et soulignée.

\section{Mémoire muséographique et histoire des musées}

Bien sûr, mettre en évidence le changement des accrochages tout au long de l'histoire d'un musée constitue aussi une façon de revendiquer la pluralité des discours et de relativiser les anciennes pratiques muséographiques. Ce constat est clair au musée national du Costa Rica, où le lambris d'or d'une salle autrefois occupée par la collection d'objets sacrés a été conservé, ainsi que des vieilles photographies montrant cet espace avec des vitrines pleines de chasubles et d'argenterie. Depuis 2008, on y trouve aussi les latrines de l'ancienne caserne qui occupait ce même lieu, désormais consacré à un parcours explicatif de l'histoire du bâtiment et de l'institution intitulé «De Cuartel a Museo » (de la prison au musée).

Une (auto)réflexion historique encore plus spécifique est maintenant présentée dans le musée Archéologique national de Madrid qui, fermé pendant de nombreuses années pour restauration, a rouvert ses portes en mars 2014. Il présente à l'étage supérieur des images et des matériaux non seulement sur les origines du musée au XIXe siècle, mais aussi sur certains épisodes de son histoire, comme l'évacuation de ses principaux trésors pendant la Guerre Civile. Il est dommage que, après cette présentation joliment intitulée «Un siècle entre vitrines » on n'ait pas poursuivi cette chronique institutionnelle jusqu'à nos jours pour la rendre plus complète et contemporaine. L'ancienne vitrine installée à l'étage principal du musée d'Ethnologie à Saragosse, rouvert en juin 2010, constitue un exemple plus modeste : une vieille casquette du gardien qui y travaillait dans les années 1950 y figure, à côté de la plaque des horaires d'ouverture affichée autrefois sur la porte d'entrée, ainsi qu'une maquette et des cartes postales anciennes montrant comment l'accrochage était organisé à cette époque, quand il portait encore le nom de museo de Ciencias Naturales... 


\section{EL MUSEO QUE FUE}

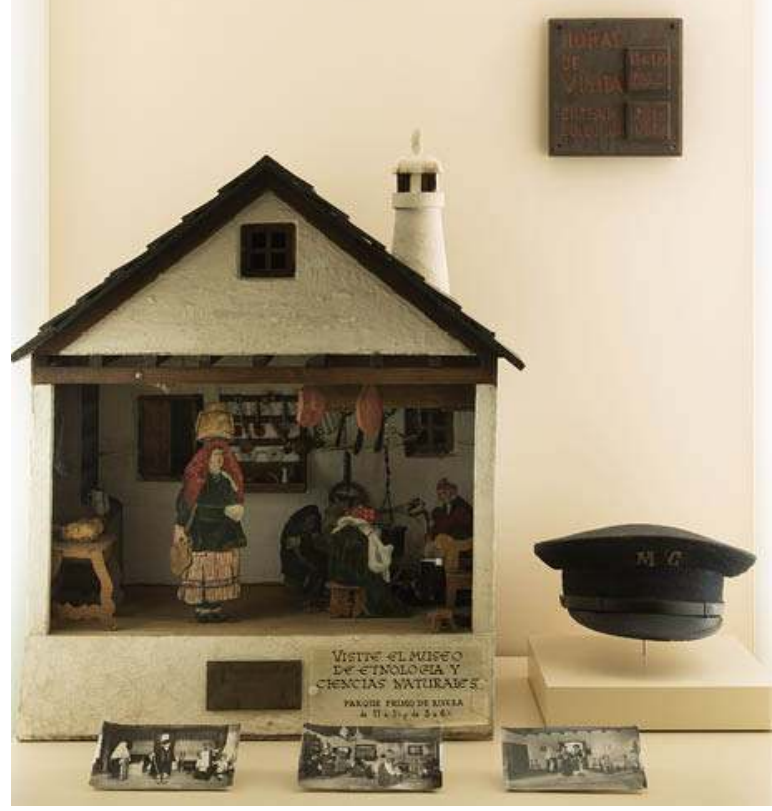

(c) Musée de Saragosse/José Garrido Lapeña

Il n'est cependant pas forcément nécessaire de retrouver des antiquités muséographiques dans les réserves d'un musée pour évoquer en quoi il était différent auparavant : il suffit de mettre dans certaines salles une photographie qui montre les anciennes présentations. À cet égard, un des exemples les plus impressionnants se trouve au cœur du musée national d'Anthropologie du Mexique. Les touristes y prennent maintenant des photographies d'eux-mêmes devant la réplique de la couronne de plumes de Moctezuma dans une vitrine à hauteur de tête ; mais, à côté est présentée une photographie ancienne montrant des visiteurs regardant respectueusement cette même coiffure - auparavant présentée dans une installation révérencieuse (les visiteurs étaient pratiquement obligés de s'agenouiller). Il faut savoir qu'à cette époque cette réplique a provoqué un grand nombre de protestations nationalistes revendiquant la restitution de la couronne originale, exposée par un musée autrichien.

Dans la collection d'antiquités étrusques du musée du Vatican, on trouve aussi sur les cimaises des panneaux des photographies montrant comment les bronzes et statues étaient entassés dans le musée il y a un siècle. Le contraste est particulièrement saisissant avec les vitrines actuelles, modernes et ne présentant que quelques objets drastiquement sélectionnés. Le danger de cette antithèse visuelle serait d'induire l'opposition manichéenne à laquelle la publicité nous a habitués, en comparant une image de quelqu'un « avant » sacrément laid, et devenu beaucoup plus attrayant « après ». Mais il suffit de multiplier les images pour donner une impression beaucoup moins simpliste et faire réfléchir les visiteurs aux fluctuations des muséographies et aux tournants de l'évolution de l'institution muséale, comme au musée Victor Balaguer à Vilanova (Barcelone) qui, pour marquer le 125e anniversaire de l'institution, montre depuis une 
série d'images comment la muséographie a changé à des moments différents. Il serait encore mieux de compléter ces contrastes photographiques avec un texte qui élimine toute tentation d'y voir un quelconque "progrès». Les choix et les aménagements muséographiques passés ne sont pas inférieurs à ceux d'aujourd'hui et méritent la même estime et le même respect que ceux d'aujourd'hui. C'est déjà le cas de la muséographie de la « salle des drapeaux » du musée national de Bogotá ; un panneau d'information, près de l'entrée, présente une description des changements dans les présentations tout au long de l'histoire du musée sous le titre : "Qu'est-ce qui a été présenté dans cette salle? ?. Le texte est encadré par deux photographies historiques d'accrochages anciens contrastant avec la muséographie contemporaine. Il s'agit, par conséquent, de documenter l'histoire de chaque musée en montrant les modifications et les indécisions, pour faire comprendre au public que les choses auraient pu être muséographiées différemment.

Les théories postmodernes, et la demande de discours autoréférentiels (dans lequel le musée parle de lui-même), ont rendu les visiteurs plus enclins à réfléchir sur le propre travail du musée. En plus de découvrir le contenu du musée et ses muséographies, on veut aussi tout savoir sur les auteurs du discours muséal lui-même. Pourquoi les taire ou les cacher si l'institution en est fière? Le musée d'Histoire naturelle de Santiago du Chili, le plus ancien de ce pays, accueille désormais les visiteurs avec un montage photographique à l'entrée commémorant le centenaire de la muséologue Grete Mostny (1914-1991). On voit cette dernière, le jour de son départ en retraite, photographiée en compagnie du personnel du musée. L'histoire de la muséologie, qui vit un moment critique, dans tous les sens du terme, se tisse ainsi avec l'histoire des musées.

L'évolution de la muséographie du musée présentée par l'image dans le hall d'entrée du musée Victor Balaguer, Vilanova i la Geltrù, Barcelone.

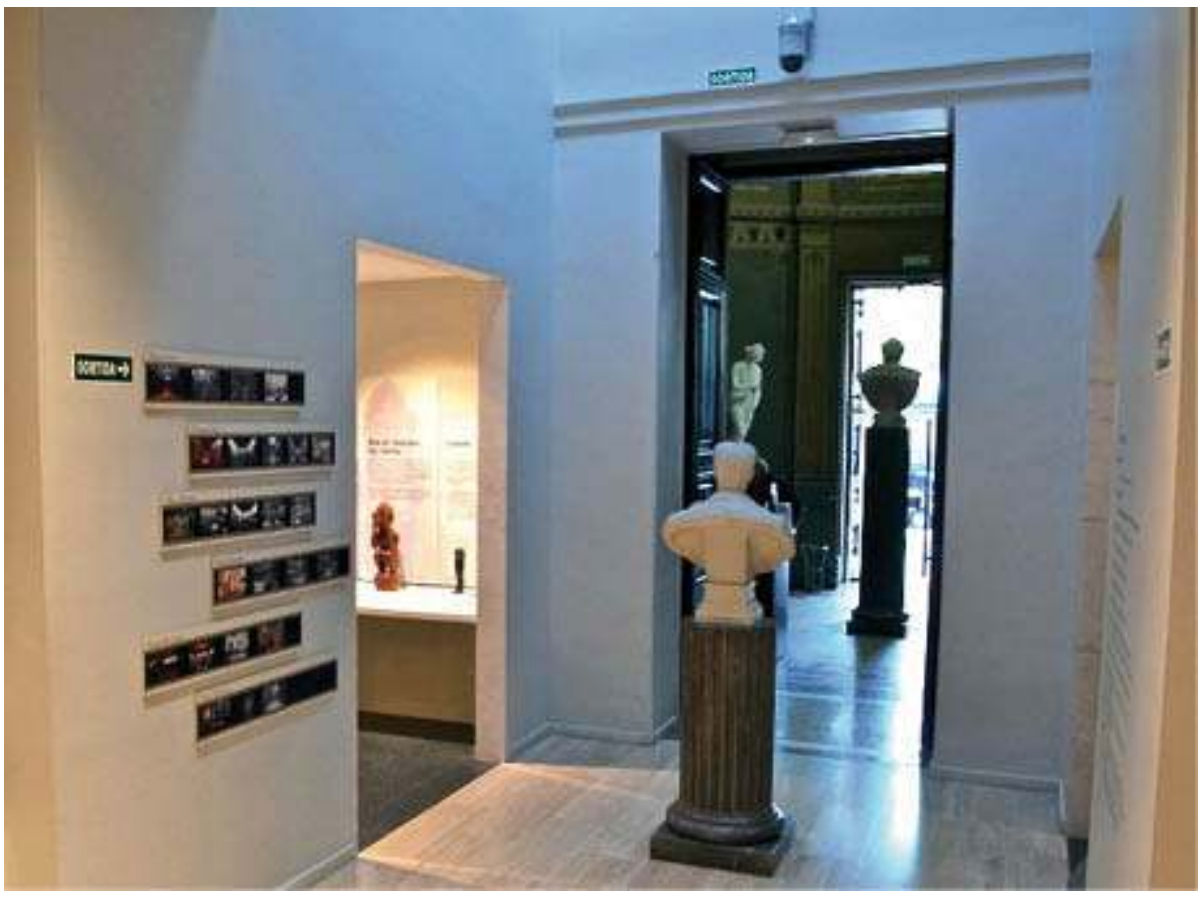

(c) Musée Victor Balaguer

Les musées négligent souvent la nature subjective des discours qu'ils construisent. Ils courent le risque de figer les objets dans des accrochages monothématiques, linéaires. 
L'enjeu pour le musée critique consiste à ne jamais cesser de remettre en question sa propre histoire, sa propre culture et même sa propre voix. Pour cela, des décisions collectives et partagées sur le mode d'accrochage, ainsi que les interprétations accessibles sur des projets ad hoc sont utiles. Mais plus encore, les idées qui naissent de l'étude conjointe des collections et du public tout comme d'un échange réciproque et continu entre les professionnels et les visiteurs sont des voies nouvelles. Chaque musée, comme une machine à remonter le temps, a la possibilité de regarder vers l'extérieur et de réinterpréter ce qu'il conserve, en incluant de nouvelles voix et diverses perspectives (parfois même celles du passé). Pour revivre dans le présent, acquérir de l'importance dans la société et permettre au visiteur de se positionner soi-même dans le monde d'aujourd'hui d'un œil réfléchi et, évidemment, critique.

\section{NOTES}

1. Hooper-Greenhill, E. (ed.) The Educational Role of the Museum. London : Routledge, 1999. Lorente, J.-P. Critical museology : Museums and exhibitions as public spaces of controversy and collective participation, Art.es, $n^{\circ} 52,2012$, pp. 98-103.

2. Un atelier a été consacré à cette thématique lors du 37e Symposium International de l'ICOFOM sur les "Nouvelles tendances de la muséologie", organisé à Paris en juin 2014, auquel ont participé les auteurs de cet article, qui nous livrent ce texte conjoint comme suite à ces deux essais destinés à être publiés dans ICOFOM Study Series en 2015 sous la direction de François Mairesse.

3. McLuhan, M., Parker, H. et Barzun, J. Le Musée non linéaire. Exploration des méthodes, moyens et valeurs de la communication avec le public par le musée. Lyon : Aléas, 2008.

4. Hein, G.-E. Learning in the Museum. London : Routledge, 1998. Falchetti, E. Costruire il pensiero scientifico in museo. Spunti e riflessioni sull'educazione scientifica nei musei delle scienze, Museologia Scientifica. $n^{\circ} 1,2007$. Grenier, C. La fin des musées ? Paris : Éditions du Regard, 2013.

5. Falk, J.-H and Dierking, L.-D. Learning from Museums : Visitor Experiences and the Making of Meaning. California: Altamira Press, 2000. Hein, G.-E. Constructivist Learning Theory, in Developing Museum Exhibition for Lifelong Learning, ed. by Gail Durbin. London : The Stationery Office, 1996, pp. 30-34. Lankford, L.-E. Aesthetic Experience in the Constructivist Museums, Journal of Aesthetic Education, 36 (2), 2002, pp. 140-153.

6. http://museodelpatrimoniomunicipal.malaga.eu, rubrique Publications

7. Science Museum : www.sciencemuseum.org.uk/whoami.aspx

8. C'est exactement ce que dit le rapport Museum 2020 qui présente l'avenir des musées. Voir aussi : Knell, S. MacLeod, J.-S. and Watson, S. (eds.) Museum Revolutions : How Museums change and are changed. Londres-New York : Routledge, 2007. Gob, A. Le musée, une institution dépassée ? Paris : Armand Collin, 2010. Sandell, R. et Nightingale, E. (ed.) Museums, equality, and social justice. Abingdon, Oxon ; New York : Routledge, 2012.

9. Pour une revendication du musée comme palimpseste de muséographies passés voir : Fehr, M. A museum and its memory : the art of recovering history, in Crane, S. Museums and Memory. Stanford : Standord University Press, 2000, pp. 35-59. La perte complète des discours muséaux communistes dans l'Europe de l'Est a été déplorée par Gamboni, D. The Destruction of Art. 
Iconoclasm and Vandalism since the French Revolution. Londres : Reaktion Books, 2007. Sur les défis de notre profession dans un monde changeant voir Janes, R.-R. Museums in a Troubled World : Renewal, Irrelevance, or Collapse? Abingdon : Routledge, 2009.

\section{RÉSUMÉS}

Qu'est-ce que la muséologie critique ? Cette contribution dresse les contours de quelques-unes des caractéristiques de ce courant particulier, telles qu'on les retrouve dans la pratique actuelle des musées, et qui visent notamment à remettre en cause la hiérarchie des savoirs entre professionnels et publics.

\section{INDEX}

Mots-clés : muséologie critique

\section{AUTEURS}

\section{JESUS PEDRO LORENTE}

professeur de Muséologie au département d'Histoire de l'Art, université de Saragosse jpl@unizar.es

\section{NICOLE MOOLHUIJSEN}

responsable du développement des expositions temporaires et des relations extérieures à Vitraria Glass + A Museum à Venise n.moolhui@gmail.com 